\title{
EL ACUERDO DE PARÍS SOBRE CAMBIO CLIMÁTICO
}

\author{
Autores: Xavier Labandeira \\ Universidad de Vigo \\ Economics for Energy \\ Pedro Linares \\ Universidad Pontificia Comillas \\ Economics for Energy
}

El pasado 12 de diciembre se aprobó por parte de la Conferencia de las Partes de la Convención Marco sobre Cambio Climático de Naciones Unidas el Acuerdo de París. El Acuerdo es un hito muy importante en la lucha contra el cambio climático, aunque quizá no tanto como nos dicen los comentaristas más triunfalistas. En este breve artículo evaluamos los elementos positivos y los no tan positivos del Acuerdo desde el punto de vista de la resolución de la externalidad global que constituye el problema del cambio climático. Para ello comenzamos recordando la magnitud del reto a la que nos enfrentamos, y posteriormente analizamos la capacidad del Acuerdo para enmarcar la respuesta necesaria. Concluimos con una reflexión sobre las implicaciones para el futuro. 


\section{LA MAGNITUD DEL PROBLEMA DEL CAMBIO CLIMÁTICO}

El cambio climático es uno de los problemas ambientales más relevantes a los que se enfrenta nuestra sociedad. Existe un consenso absolutamente generalizado entre los científicos de que el cambio climático se está produciendo, de que ya estamos comenzando a experimentar sus efectos, de que sus consecuencias serán aún más severas en el futuro, y de que el principal responsable de ello es la especie humana, a través de las emisiones de gases de efecto invernadero (GEI) asociadas a muchas de nuestras actividades.

La necesidad de actuar es además urgente: según estimaciones recientes, si mantenemos la tendencia actual de emisiones de gases de efecto invernadero, nos quedan menos de 25 años para agotar el llamado "presupuesto de carbono", es decir, la cantidad total de GEI que podemos poner en la atmósfera sin arriesgarnos a cambios peligrosos de nuestro sistema climático.

Por si esto no fuera suficiente para hacernos conscientes de la necesidad de actuar pronto frente al cambio climático, hay que recordar que este problema tiene importantes consecuencias distributivas entre países y grupos sociales, que se enfrentan a impactos de distinta magnitud con grandes diferencias en sus capacidades de adaptación. En general, el cambio climático afectará más negativamente, y será más complicado adaptarse a él, en las regiones menos desarrolladas del planeta, constituyendo pues otro elemento más de esa crisis integral que nos señala tan acertadamente el Papa Francisco en su encíclica Laudato Si.

Para poder responder de manera apropiada a esta amenaza debemos cambiar de manera muy relevante la forma en la que producimos y utilizamos la energía, así como algunos procesos industriales, y también algunas prácticas agrícolas, ganaderas, y de uso del territorio (en particular la deforestación). Para poder mantener las concentraciones de GEI en niveles aceptables parece necesario que las emisiones de gases de efecto invernadero globales alcancen su máximo en la década de 2030, y que en la segunda mitad del siglo XXI se reduzcan hasta niveles próximos a cero. Esto requiere cambios tecnológicos de gran calado, pero también, posiblemente, cambios en la forma en que realizamos distintas actividades, nos desplazamos, o en los productos que demandamos.

Y por supuesto, también será necesario adaptarnos a la subida de temperaturas o del nivel del mar, o al aumento de eventos extremos, previsibles para las próximas décadas porque, desgraciadamente, y debido a la inercia del sistema climático, ya es imposible evitar alguno de sus efectos.

Sin embargo, las actuaciones previstas no parecen estar a la altura: los compromisos para los próximos años de los países, expresados en las llamadas contribuciones nacionales (NDCs, nationally-determined contributions) sitúan el aumento de la temperatura esperada para finales de siglo cerca de los $3^{\circ} \mathrm{C}$, y los escenarios futuros como los planteados por la Agencia Internacional de la Energía o BP 
nos llevan hasta los $3,5^{\circ} \mathrm{C}$, lejos del nivel considerado seguro de $2{ }^{\circ} \mathrm{C}$. Algunos podrían argumentar que, en el caso de las empresas energéticas, o incluso en el de la AIE, estos escenarios tratan de preservar el status-quo, es decir, que tienen un papel político. Pero eso no nos debe despistar: cualquier lectura imparcial de la situación nos lleva a concluir que lograr los objetivos de $2^{\circ} \mathrm{C}$ (y no digamos el de $1,5^{\circ} \mathrm{C}$ ) es muy difícil. Un ejemplo es la evaluación de la credibilidad de las promesas para París de los países G20, elaborada por el Grantham Institute ${ }^{1}$.

Parte del problema es por supuesto la dificultad del cambio tecnológico y de comportamientos ya mencionados. Estos cambios además pueden resultar más costosos para unos países que para otros, en función de su acceso desigual a las distintas tecnologías o modelos de desarrollo.

Pero otra parte viene del hecho de que, frente a una externalidad global de este tipo, el incentivo a ir de polizón ("free ride", según los anglosajones) es muy fuerte. Dicho de otra forma, hay pocos beneficios en la aplicación unilateral de medidas contra el cambio climático no sólo por la pérdida de competitividad relativa que supone para un país o grupo de países la aplicación de este tipo de políticas, sino también por el riesgo de fuga de emisiones, es decir, el hecho de que las actividades productivas (y emisoras de GEI) se desplacen simplemente de un lugar a otro para evitar la regulación, sin reducirse o incluso aumentando. Así, los verdaderos beneficios de una política climática sólo se manifiestan cuando ésta es aplicada de forma generalizada, extendiéndose a todos los sectores y países responsables de las emisiones de GEI.

Por último, hay que recordar que también las políticas climáticas tienen efectos distributivos importantes. Además de incentivar el desarrollo de nuevas tecnologías, modelos de negocio, o modelos de desarrollo, estas políticas, si se aplican como es necesario, también crearán perdedores, principalmente entre las economías y empresas dedicadas a la explotación, transporte, conversión y distribución de combustibles fósiles. Estos potenciales perdedores pueden constituir muy poderosas minorías de bloqueo en contra del interés general.

Este es el contexto en el que debe analizarse el Acuerdo de París, un acuerdo que debería coordinar los esfuerzos internacionales para reducir significativamente las emisiones de GEI en las próximas décadas, y además atender a todas las cuestiones distributivas suscitadas anteriormente. Un acuerdo de evidente complejidad, pues. Veamos en qué medida ha logrado estos objetivos.

\section{UNA VALORACIÓN DEL ACUERDO DE PARÍS}

Un acuerdo complejo como éste puede analizarse desde distintas perspectivas. Una forma muy ingeniosa de formularlo es la de George Monbiot que decía que

${ }^{1}$ http://www.lse.ac.uk/GranthamInstitute/wp-content/uploads/2016/01/Averchenkova-and-Bassi-2016.pdf 
"en comparación con lo que podría haber sido, es un milagro; en comparación con lo que debería haber sido, es un desastre". El acuerdo puede verse pues como un vaso medio lleno o como un vaso medio vacío.

En general, la recepción del Acuerdo ha sido positiva. Las organizaciones ecologistas más representativas, por ejemplo, recibieron con moderada satisfacción el resultado (véase por ejemplo el comunicado de Greenpeace ${ }^{2}$ ). Bloomberg, que inicialmente habló de fiasco, posteriormente se unió ${ }^{3}$ a la interpretación estándar: un paso adelante.

Pero quizá es especialmente sorprendente, al revisar las reacciones, la unanimidad entre los (principalmente) economistas académicos que trabajan en este campo y han hecho pública su opinión. Ottmar Edenhofer, ex-presidente del grupo de trabajo 3 del IPCC, define el acuerdo como un cambio fundamental que hay que aprovechar ${ }^{4}$. Robert Stavins solo ve aspectos positivos en lo acordado $^{5}$ y Nicholas Stern habla de un punto de inflexión histórico. Muy indicativo es también el tono optimista de David Victor, muy poco dado habitualmente a este tipo de interpretaciones, que sugiere que el acuerdo conseguido es superior a aproximaciones basadas en obligaciones y sanciones ${ }^{7}$. Carlos Duarte, uno de los científicos españoles más reputados en este campo, califica el acuerdo con un 9 sobre 10 por su aproximación descentralizada, el fin de la influencia de los climaescépticos, y su promoción implícita de tecnologías bajas en carbono. Un debate reciente $^{8}$ organizado en FSR Climate entre tres académicos muy representativos en esta área en Europa (Carlo Carraro), China (Xiliang Zhang) y EEUU (Joe Aldy) y una audiencia global muy participativa, mostró de nuevo una gran unanimidad en los avances de París, con escasas matizaciones.

¿Y nuestra valoración? Pues similar: en general positiva, pero con matizaciones de calado.

Comencemos con una revisión breve de los elementos fundamentales del acuerdo. Sin entrar en los detalles, que se pueden consultar en la web del UNFCCC 9 , sí podemos indicar lo nuevo y lo no tan nuevo. Lo nuevo es sin duda la cobertura, que alcanza a todos los países dispuestos a mitigar sus emisiones, su carácter voluntarista, y el énfasis en la verificación y transparencia. Lo no tan nuevo tiene

\footnotetext{
${ }^{2}$ http://www.greenpeace.org/eu-unit/en/News/2015/Paris-climate-deal-EU-performance/

${ }^{3}$ http://www.bloomberg.com/news/articles/2015-12-12/landmark-climate-change-agreement-hailedas-leap-for-mankind-

${ }^{4}$ https://www.mcc-berlin.net/en/topnews/the-relevance-of-the-paris-agreement-for-the-world.html

5 http://www.robertstavinsblog.org/2015/12/12/paris-agreement-a-good-foundation-for-meaningfulprogress/

${ }^{6}$ http://internacional.elpais.com/internacional/2015/12/21/actualidad/1450716682_907428.html

${ }^{7}$ http://www.economist.com/node/18557392

${ }^{8}$ http://fsr.eui.eu/event/online-debate-cop21-results-and-perspectives/

${ }^{9}$ http://unfccc.int/resource/docs/2015/cop21/spa/109s.pdf
} 
que ver con las características abajo/arriba, los objetivos ambientales exógenos, la importancia de la adaptación y el establecimiento de fondos distributivos que permitan visualizar las responsabilidades comunes pero diferenciadas, todo ello originado en cierta medida en la teóricamente fracasada COP de Copenhague.

El acuerdo parte pues de toda la historia de negociaciones climáticas internacionales, y sobre ella introduce algunos elementos que han permitido finalmente que todos los países se unan a él. La presidencia francesa, heredera de su tradición nacional en el arte de la diplomacia, ha vuelto a demostrar sus dotes. Pero no por sacar un acuerdo por arte de magia de donde no lo había, sino por ser capaz de convencer a todos de que había elementos en los que todos podían estar de acuerdo. El precio que se paga por ello, claro, es sacar del acuerdo todo lo controvertido, y dejar lo demás por definir. Pero seguramente esta era la única posibilidad real de acuerdo, como decía Monbiot. En este sentido, es un acuerdo realista, como ya reclamábamos desde $2011^{10}$.

En cualquier, caso, el acuerdo es un claro paso adelante. Por primera vez, todos los países se comprometen a reducir sus emisiones de GEI, y a hacerlo de forma transparente para el resto. Además, el requerir (en uno de los pocos requerimientos reales) las contribuciones nacionales (NDCs) revisables cada 5 años puede dar un poco de fuerza moral, de presión, a los países, y ampararles frente a los escépticos domésticos. En este sentido, el Acuerdo supone un impulso importante para las dinámicas que ya estaban en marcha en muchos países, y consagra el papel central de las NDCs. Finalmente, también es enormemente positivo el cambio de dinámica geopolítica que supone, al superar la ya anticuada división del Protocolo de Kyoto entre países Anexo I (OCDE) y no-Anexo I (que incluía entre otros a China o México).

Sin embargo, tampoco podemos caer en el triunfalismo desatado en los días inmediatamente posteriores al acuerdo. Este Acuerdo en sí mismo no salvará al clima. Primero, porque está lejos de la solución óptima, y segundo, porque todavía no sabemos si el Acuerdo funcionará.

En efecto, hay serias dudas de que el marco propuesto en París sea efectivo para mantener las emisiones dentro de los límites necesarios. Es cierto que, por primera vez en la historia de la negociación climática, el Acuerdo incluye instrumentos de corrección dinámica, de revisión de objetivos. Y además también introduce elementos esperanzadores. En primer lugar da flexibilidad a los emisores, permitiéndoles ser más ambiciosos al ajustar sus necesidades (por ejemplo, reducción de dependencia energética y/o de contaminación local) y capacidades con sus contribuciones, mitigando así el problema free-rider. En segundo lugar puede permitir la creación de coaliciones de grandes emisores (recordemos que menos de diez países, incluyendo como tal a la UE, son responsables de aproximadamente dos tercios de

\footnotetext{
${ }^{10}$ http://elpais.com/diario/2011/12/03/sociedad/1322866802_850215.html
} 
las emisiones globales) que ajusten coordinadamente sus contribuciones voluntarias porque el Acuerdo reducirá sus preocupaciones sobre efectos competitivos y la fuga de emisiones. Pero no soluciona en sí mismo el problema del polizón implícito en la cuestión del cambio climático, ni asegura la reducción de emisiones necesaria.

Como ya decíamos, las contribuciones nacionales no nos llevan más allá de los $3^{\circ} \mathrm{C}$. Y estas contribuciones tienen sus propios problemas. Para comenzar, muchas de las estrategias nacionales están basadas en la eficiencia energética: es barata, y es poco intrusiva. Pero también es muy difícil de lograr, como la experiencia nos indica.

El otro factor esencial es el precio de los combustibles fósiles. Mientras que el carbón y el petróleo sigan siendo tan baratos, es difícil que el mercado vaya a promover su sustitución. De nuevo, se podría pensar que, al menos en el caso del petróleo, los bajos precios actuales son temporales y que su precio se volverá a recuperar al menos hasta los 70-80 dólares por barril....pero esto no arregla mucho: simplemente por dinámica de mercado, si queremos abandonar el petróleo para descarbonizar el transporte, su demanda baja y también su precio. Lo mismo sucede (de hecho ya está sucediendo) con el carbón. Es decir, que los bajos precios de los fósiles van a estar indisolublemente unidos a esta transición hacia los sistemas energéticos descarbonizados, y por tanto no podemos confiar en el diferencial de precios para reducir su uso.

Salvo, claro, que hagamos algo, básicamente corregir la externalidad negativa asociada a los fósiles. Pero de nuevo, una cosa es la teoría y otra la práctica. Los precios del $\mathrm{CO} 2$ en los mercados de emisiones siguen bajos, y no parece que vayan a llegar a los niveles necesarios (sobre los 150 euros/t, según algunas estimaciones propias que coinciden con las de la Agencia Internacional de la Energía). Antes bien, muchos países siguen subvencionando de distintas formas la producción y consumo de las energías fósiles.

$\mathrm{Si}$ a esto le sumamos que a la nuclear, al menos en la OCDE, no le acaban de salir las cuentas; que los biocombustibles siguen presentando grandes problemas en términos de utilización del territorio (el llamado ILUC), y que su desarrollo es muy frágil en cuando se reconsideran las políticas de apoyo; y que el CCS ni está ni se le espera (no tanto por el desarrollo tecnológico, sino más bien por la enorme complejidad de su almacenamiento), parece evidente que las contribuciones nacionales previstas tienen muchos retos internos por resolver.

A este respecto, es interesante comentar el papel reducido que han jugado en los debates y en el propio acuerdo los precios por emitir GEI. Aunque evidentemente la batería de medidas necesarias para reducir las emisiones de GEI debe ser muy amplia ${ }^{11}$, el contar con una señal de precio es fundamen-

${ }^{11}$ Fischer, C., Torvanger, A., Shrivastava, M., Sterner, T., y Stigson, P., "How should Support for Climate-friendly Technologies be Designed?" Ambio (núm. 41, sup. 1, 2012), pp. 33-45. 
tal, tanto para incentivar los comportamientos adecuados como para conseguir recursos para los fondos de adaptación o mitigación, o para compensar a los perdedores del proceso. Nordhaus ${ }^{12}$ recientemente proponía por ejemplo la creación de clubes en torno a un precio sin cláusulas penalizadoras, como instrumento para una actuación coordinada. Por ello, resulta sorprendente que no se mencione explícitamente en el acuerdo, más allá de un reconocimiento de la posibilidad de utilizar mercados de emisiones en un artículo específico.

El Acuerdo tampoco soluciona por sí mismo los problemas distributivos que avanzábamos en nuestro diagnóstico. Aunque sí introduce una distinción implícita entre mitigación de GEI en términos absolutos para los países desarrollados y en términos relativos (respecto al business-as-usual) para las economías emergentes y en desarrollo, el debate sobre 'pérdida y daño' se ha resuelto con el reconocimiento explícito de que los responsables históricos de las concentraciones atmosféricas de GEI no están sujetos al pago de indemnizaciones por daños sufridos. También se mantiene el papel de los fondos para facilitar la mitigación y la adaptación en los países en vías de desarrollo. Pero su cuantía, 100.000 millones de dólares por año, parece insuficiente en relación a las necesidades evaluadas ${ }^{13}$. Y tampoco concreta quién tiene que aportar este dinero.

De hecho, un grupo de científicos anglosajones acaba de publicar una carta ${ }^{14}$ en la que se afirma que el Acuerdo puede ser contraproducente porque se ha lanzado una falsa señal de solución cuando su integridad ambiental es muy débil y, según ellos, aboca a la humanidad hacia la geo-ingeniería, una solución con múltiples problemas.

En este sentido, el Acuerdo debe interpretarse solo como un primer paso hacia una solución más eficiente y ambiciosa que permita responder adecuadamente a los inmensos desafíos en el ámbito de la mitigación de emisiones. Para ello serán fundamentales las próximas COPs (que han de pasar de lo generalista a lo específico) y la puesta en marcha y ajuste de las contribuciones voluntarias de los países. Hace falta desarrollar todos los sistemas de reporte y verificación, dotar los fondos de adaptación y de transferencia de tecnología, y sobre todo, diseñar las políticas nacionales para alcanzar los objetivos propuestos en las NDCs e ir más allá, ya que, como decíamos, las NDCs no bastan para alcanzar un objetivo seguro de calentamiento global.

\footnotetext{
${ }^{12}$ Nordhaus, M., "Climate Clubs: Overcoming Free-Riding in International Climate Policy”, American Economic Review (núm. 5, 2015), pp. 1339-1370.

${ }^{13}$ OCDE, La Financiación para el Clima en 2013-14 y el Objetivo de los 100.000 millones de Dólares, OCDE y CPI, 2015.

$14 \mathrm{http} / /$ www.independent.co.uk/environment/climate-change/cop21-paris-deal-far-too-weak-toprevent-devastating-climate-change-academics-warn-a6803096.html
} 


\section{LAS IMPLICACIONES DEL ACUERDO PARA EL FUTURO}

A pesar de sus limitaciones, el Acuerdo sí señala y refuerza una serie de dinámicas que ya se estaban produciendo respecto al fenómeno del cambio climático. Muchos países, y en especial los grandes emisores, tienen interés en reducir sus emisiones, por causas directas o indirectas. Aunque quizá no al nivel de ambición necesario, fenómenos como la contaminación local, como la seguridad energética, o como el avance tecnológico, ya están llevando a algunos países a plantearse la necesidad de reducir sus emisiones de GEI, y, para ello, de transformar sus sistemas energéticos.

En este sentido, la cobertura mediática tan significativa que ha tenido el acuerdo, o el apoyo político sin precedentes (nunca se habían juntado tantos jefes de estado o de gobierno en un acto similar) puede añadir ímpetu a este tipo de transformaciones. Así, el Acuerdo refuerza la idea de un escenario futuro en el que se desaparecen las emisiones antropogénicas de gases de efecto invernadero, y al que tendrán que adaptarse nuestras sociedades y empresas.

Sin embargo, la carrera hacia este futuro sin emisiones de GEI acaba de empezar. Hay que ser conscientes de la magnitud de los retos a los que nos enfrentamos, y que no se han resuelto con este Acuerdo.

Las políticas nacionales (o europeas, en su caso) tienen un papel central a este respecto. Ni los mercados ni el desarrollo tecnológico por sí solo nos van a arreglar los problemas si no les ayudamos y guiamos. El futuro sin carbono pasa por una regulación pública que dé las señales necesarias para el cambio, de forma coherente. Así, parece evidente que deberán desaparecer las subvenciones a los combustibles fósiles, por otra parte ineficientes económica y socialmente. También habrá que armonizar las políticas fiscales o urbanísticas con los objetivos buscados. Y, más allá de los precios, dar también señales de largo plazo que eviten inversiones contraproducentes, no alineadas con esfuerzos de mitigación y adaptación necesarios.

Las empresas también tendrán un papel central que jugar. Deberán ser las ejecutoras de los procesos de innovación necesarios, en colaboración con los gobiernos; deberán entender y responder a los nuevos modelos de negocio que crecientemente exigen los consumidores; y sobre todo, deberán adaptarse a etapas de precios bajos de los combustibles fósiles y de eventual abandono de los mismos.

También las universidades y centros de investigación como el nuestro ${ }^{15}$ pueden contribuir significativamente, analizando tecnologías, escenarios y políticas de forma rigurosa e independiente, y proponiendo soluciones efectivas, eficientes y justas a nuestros políticos y empresas. De hecho, muchos de estos centros están

\footnotetext{
${ }^{15}$ www.eforenergy.org
} 
ya centrando sus esfuerzos, o identificando como líneas prioritarias, aquellas relacionadas con la energía y la sostenibilidad.

El Acuerdo de París representa un considerable paso adelante y un innegable éxito diplomático en la lucha contra el cambio climático. Sin embargo son muchos los interrogantes que persisten y solo seremos capaces de ver los efectos de este acuerdo dentro de unos años, a partir de sus desarrollos en futuros procesos de negociación internacional y, sobre todo, de la aplicación de políticas climáticas nacionales para el cumplimiento de los objetivos voluntarios establecidos. En cualquier caso, nos marca una línea clara de evolución para nuestras economías y sistemas productivos, a la que todos debemos adaptarnos.

Madrid, 14 de abril de 2016 